\title{
A Test for the Rapid Detection of the Cefazolin Inoculum Effect in Methicillin-Susceptible Staphylococcus aureus
}

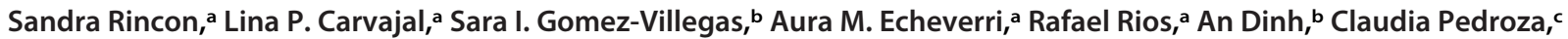

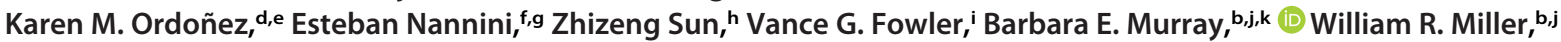 \\ (D) Timothy Palzkill, ${ }^{h}$ Lorena Diaz, ${ }^{a}$ Cesar A. Arias, ${ }^{\text {bj, }, k, l}$ (D) Jinnethe Reyes ${ }^{a}$

\begin{abstract}
aMolecular Genetics and Antimicrobial Resistance Unit, International Center for Microbial Genomics, Universidad El Bosque, Bogota, Colombia
bCenter for Antimicrobial Resistance and Microbial Genomics, McGovern Medical School, University of Texas Health Science Center, Houston, Texas, USA

‘Center for Clinical Research and Evidence-Based Medicine, McGovern Medical School, University of Texas Health Science Center, Houston, Texas, USA

dE.S.E. Hospital Universitario San Jorge de Pereira, Risaralda, Colombia

eUniversidad Tecnológica de Pereira, Risaralda, Colombia

fFacultad de Ciencias Médicas, Universidad Nacional de Rosario, Rosario, Argentina

IInstituto de Inmunología Clínica y Experimental de Rosario, CONICET, Rosario, Argentina

nBaylor College of Medicine, Houston, Texas, USA

'Division of Infectious Diseases, Department of Medicine, Duke University, Durham, North Carolina, USA

kDepartment of Microbiology and Molecular Genetics, McGovern Medical School, University of Texas Health Science Center, Houston, Texas, USA

'Center for Infectious Diseases, School of Public Health, University of Texas Health Science Center at Houston, Houston, Texas, USA
\end{abstract} \\ jDivision of Infectious Diseases, Department of Internal Medicine, McGovern Medical School, University of Texas Health Science Center, Houston, Texas, USA
}

Sandra Rincon and Lina P. Carvajal contributed equally to the manuscript. Author order was determined in order of increasing seniority.

Cesar A. Arias and Jinnethe Reyes contributed equally to the manuscript.

ABSTRACT The cefazolin inoculum effect (CzIE) has been associated with therapeutic failures and mortality in invasive methicillin-susceptible Staphylococcus aureus (MSSA) infections. A diagnostic test to detect the CzIE is not currently available. We developed a rapid ( $\sim 3 \mathrm{~h}$ ) CzIE colorimetric test to detect staphylococcal- $\beta$-lactamase (BlaZ) activity in supernatants after ampicillin induction. The test was validated using 689 bloodstream MSSA isolates recovered from Latin America and the United States. The cefazolin MIC determination at a high inoculum $\left(10^{7} \mathrm{CFU} / \mathrm{ml}\right)$ was used as a reference standard (cutoff $\geq 16 \mu \mathrm{g} / \mathrm{ml}$ ). All isolates underwent genome sequencing. A total of 257 (37.3\%) of MSSA isolates exhibited the CZIE by the reference standard method. The overall sensitivity and specificity of the colorimetric test was $82.5 \%$ and $88.9 \%$, respectively. Sensitivity in MSSA isolates harboring type A BlaZ (the most efficient enzyme against cefazolin) was $92.7 \%$ with a specificity of $87.8 \%$. The performance of the test was lower against type $B$ and $C$ enzymes (sensitivities of $53.3 \%$ and $72.3 \%$, respectively). When the reference value was set to $\geq 32 \mu \mathrm{g} / \mathrm{ml}$, the sensitivity for isolates carrying type A enzymes was $98.2 \%$. Specificity was $100 \%$ for MSSA lacking blaZ. The overall negative predictive value ranged from $81.4 \%$ to $95.6 \%$ in Latin American countries using published prevalence rates of the CzIE. MSSA isolates from the United States were genetically diverse, with no distinguishing genomic differences from Latin American MSSA, distributed among 18 sequence types. A novel test can readily identify most MSSA isolates exhibiting the CzIE, particularly those carrying type A BlaZ. In contrast to the MIC determination using high inoculum, the rapid test is inexpensive, feasible, and easy to perform. After minor validation steps, it could be incorporated into the routine clinical laboratory workflow.

KEYWORDS CzIE rapid test, BlaZ, cefazolin inoculum effect, high inoculum

he he clinical burden of infections caused by methicillin-susceptible Staphylococcus aureus (MSSA) is significant (1-3). In some parts of the world, the incidence of
Citation Rincon S, Carvajal LP, Gomez-Villegas SI, Echeverri AM, Rios R, Dinh A, Pedroza C, Ordoñez KM, Nannini E, Sun Z, Fowler VG, Murray BE, Miller WR, Palzkill T, Diaz L, Arias CA Reyes J. 2021. A test for the rapid detection of the cefazolin inoculum effect in methicillinsusceptible Staphylococcus aureus. J Clin Microbiol 59:e01938-20. https://doi.org/10 $.1128 /$ JCM.01938-20.

Editor Carey-Ann D. Burnham, Washington

University School of Medicine

Copyright $\odot 2021$ American Society for Microbiology. All Rights Reserved.

Address correspondence to Cesar A. Arias, caa22@cantab.net, or Jinnethe Reyes, reyesjinnethe@unbosque.edu.co.

Received 25 July 2020

Returned for modification 20 September 2020

Accepted 23 January 2021

Accepted manuscript posted online

3 February 2021

Published 19 March 202 
invasive infection caused by MSSA is higher than that caused by methicillin-resistant $S$. aureus (MRSA) (4). Cefazolin, a first generation cephalosporin, is now used by many as the preferred therapy for MSSA infections because several studies have suggested that it is safer, easier to administer, and as effective as anti-staphylococcal penicillins (ASP) (5-12). In some regions of the world, it is the only preferred antibiotic that is routinely available to treat invasive MSSA infections (13).

The cefazolin inoculum effect (CzIE) is characterized by a marked increase in the MIC when a high bacterial inoculum, typically at least $10^{7} \mathrm{CFU} / \mathrm{ml}$, is used to determine the MIC value. The CzIE has been associated with therapeutic failures and increased mortality in deep-seated infections (13-15), and appears to vary in frequency from 3 to $20 \%$ of MSSA isolates from Asia (16-18) and the United States (19-21) up to 30 to 54\% in MSSA isolates from Latin America $(13,22,23)$.

The mechanistic basis of the CzIE involves the staphylococcal $\beta$-lactamase (BlaZ) enzymes, which belong to the molecular class $A$ and have been classified into four types (A to D) (24). BlaZ types $A$ and $C$ have been the most common types associated with the CzIE $(13,16-23)$. The gold standard for identification of the CzIE is by determining cefazolin MICs at high inoculum (MIC-HI) by broth microdilution (21). However, this procedure is technically difficult to implement in clinical laboratories. Nitrocefin is a chromogenic cephalosporin without antimicrobial properties that is widely used for phenotypic detection of $\beta$-lactamases in microorganisms. BlaZ hydrolyzes the $\beta$-lactam ring of nitrocefin, resulting in a distinctive color change (yellow to red) (25).

Taking advantage of this chromogenic substrate, we showed in preliminary assays that nitrocefin rapidly and accurately identified the strains that display the CzIE by detecting the presence of staphylococcal $\beta$-lactamase in bacterial supernatants after they were induced with ampicillin (26). Indeed, we observed significant differences $(P<0.001)$ in the hydrolysis of nitrocefin after $30 \mathrm{~min}$ by supernatants of $S$. aureus TX0117 (14) (positive control for the CzIE) compared to the supernatant of strains that did not exhibit the CZIE (TX0117c and ATCC 29213) (Fig. S1 in the supplemental material). Of note, the hydrolysis of nitrocefin was also readily detectable by visual inspection. Thus, these findings suggested that the CzIE was highly correlated with the presence of BlaZ in the extracellular milieu (26). Here, we describe a novel rapid colorimetric test that uses the chromogenic cephalosporin nitrocefin to detect the strains that display the CzIE in a cohort of bloodstream MSSA isolates recovered from Latin America and the United States (25).

\section{MATERIALS AND METHODS}

Bacterial isolates, typing of $\boldsymbol{\beta}$-lactamase, and phylogenetic analysis. Two sets of MSSA isolates were included in the study. The first set $(n=640)$ consists of isolates collected as part of a multicenter surveillance study of bloodstream infections in nine countries of Latin America (2011 to 2014) and, more recently (2018 to 2019), from one hospital in Pereira, Colombia (1, 23). The second set includes an archived collection of $S$. aureus bloodstream isolates $(n=49)$ recovered from the United States as part of two repositories (the Duke University repository and the International Collaboration on Endocarditis [ICE] cohort study) (21). The isolates recovered from Latin America were sent to our reference laboratory in Bogota, Colombia. Isolates collected in the United States were sent to and stored in Houston, TX (University of Texas [UT] Health Center for Antimicrobial Resistance and Microbial Genomics).

Typing of the staphylococcal $\beta$-lactamase was performed through whole-genome sequencing using BLASTX searches against S. aureus BlaZ ATCC 29213 (GenBank accession ODV53133.1). We classified BlaZ based on the amino acid residues at positions 128 and $216(27,28)$. Sequence types (ST) and clonal complexes (CC) were assigned according to the PubMLST database (29). The genetic relationship among the isolates was investigated by a core genome phylogenetic analysis. The core genome was defined using Roary v3.13.0, selecting the orthogroups in $95 \%$ of the analyzed genomes (30). Orthologous nucleotide sequences, defined by Roary, were aligned with the MUSCLE algorithm and concatenated to construct a phylogenetic matrix (31). Phylogeny was reconstructed with RAxML v8.2.11 using the GTRGAMMA and 100 rapid bootstrap replicates (32). Tree visualization and annotation was performed using the online tool Interactive Tree of Life v5.5.1 (https://itol.embl.de) (33).

Determination of the cefazolin inoculum effect. The cefazolin inoculum effect (CzIE) was evaluated by broth microdilution using standard and high bacterial inocula $\left(5 \times 10^{5} \mathrm{CFU} / \mathrm{ml}\right.$ and $5 \times 10^{7}$ CFU/ml, respectively) $(22,34)$. Cefazolin MIC assessments were performed in triplicate and evaluated by three independent investigators. Controls included S. aureus TX0117, a strain that harbors a type A BlaZ and exhibits the CzIE. This strain was recovered from a patient with endocarditis and failure of cefazolin (14). S. aureus strain ATCC 29213 harbors type A BlaZ but does not exhibit the cefazolin inoculum effect, 
A

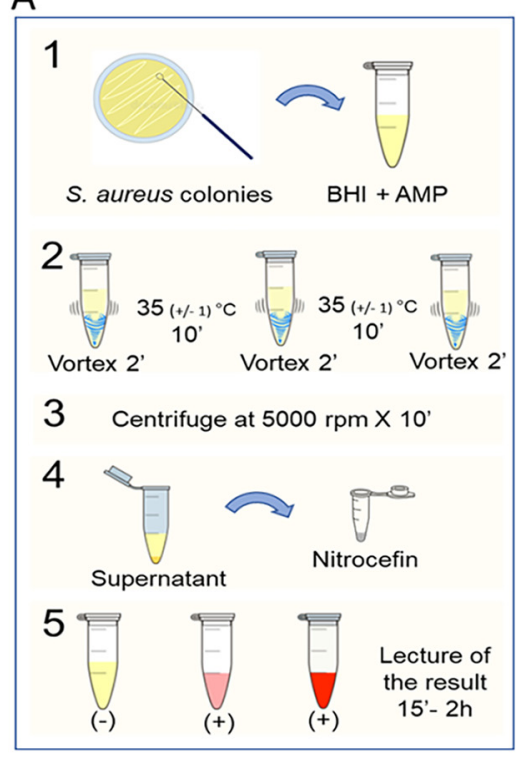

B

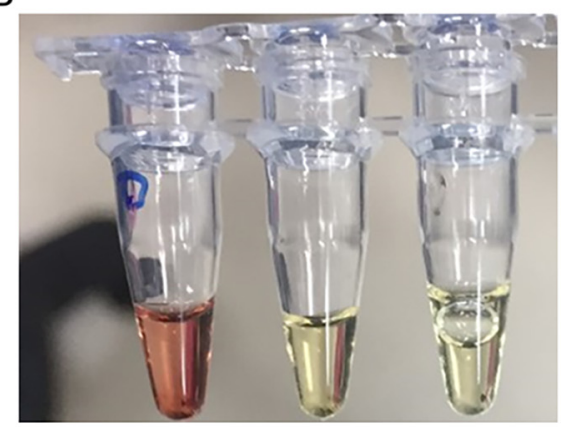

FIG 1 Methodology of the CzIE rapid test. (A) Methodology of the rapid test (as described in the Materials and Methods section). (B) S. aureus quality controls. Tubes from left to right correspond to MSSA TX0117 (BlaZ-positive exhibiting the CZIE), ATCC 29213 (BlaZ-positive lacking the CzIE), and ATCC 25923 (BlaZ-negative lacking the CzIE). Change of color to red indicates a positive result (presence of CzIE), whereas a yellow color is suggestive of a negative result.

and is widely used as a quality control strain for in vitro susceptibility testing. In addition, we also included a MSSA strain that does not possess BlaZ (ATCC 25923). The CzIE was defined as a MIC of $\geq 16 \mu \mathrm{g} / \mathrm{ml}$ at high inoculum and of $\leq 8 \mu \mathrm{g} / \mathrm{ml}$ at standard inoculum (21).

Rapid colorimetric test to detect the CzIE. Figure 1 shows technical details on the test. Nitrocefin was prepared as a stock solution at a concentration of $5 \mathrm{mg} / \mathrm{ml}$ in dimethyl sulfoxide (DMSO) (SigmaAldrich, USA) and then diluted to $400 \mu \mathrm{M}$ in phosphate-buffered saline (PBS) at $0.1 \mathrm{M}, \mathrm{pH} 7.0$. The solution was preserved in a foil-wrapped tube at $4^{\circ} \mathrm{C}$. S. aureus strains were cultured in brain heart infusion $(\mathrm{BHI})$ agar (Oxoid Limited, UK). Following incubation for 18 to $24 \mathrm{~h}$ at $37^{\circ} \mathrm{C}$, morphologically similar colonies were selected with a 1- $\mu$ l calibrated sterile loop. The loop was inoculated into a 2-ml tube containing $1 \mathrm{ml}$ of $\mathrm{BH}$ broth with ampicillin ( $150 \mu \mathrm{g} / \mathrm{ml}$, freshly prepared). The suspension was vortexed vigorously for $2 \mathrm{~min}$ to ensure homogenization and incubated at $35 \pm 1{ }^{\circ} \mathrm{C}$ for $10 \mathrm{~min}$. After incubation, the suspension was vortexed again for $2 \mathrm{~min}$. Then, a second incubation step of $10 \mathrm{~min}$ at $35 \pm 1^{\circ} \mathrm{C}$ followed by vortexing ( $2 \mathrm{~min}$ ) was carried out. The two-step procedure permits extracellular $\beta$-lactamase to be homogeneously distributed in the broth. Next, the solution was centrifuged at 5,000 rpm for $10 \mathrm{~min}$ (room temperature). While avoiding aspiration of the bacterial cell pellet, $25 \mu \mathrm{l}$ of the supernatant was transferred into a $0.2-\mathrm{ml}$ tube containing $25 \mu \mathrm{l}$ of freshly dispensed nitrocefin solution $(400 \mu \mathrm{M})$. The tubes were incubated at room temperature $\left(15\right.$ to $25^{\circ} \mathrm{C}$, mean of $22^{\circ} \mathrm{C}$ ) and protected from light, followed by visual inspection to monitor color change at 15, 30, $45 \mathrm{~min}, 1 \mathrm{~h}$, and $2 \mathrm{~h}$. A positive result was interpreted as any change in color from yellow to red from $15 \mathrm{~min}$ to $2 \mathrm{~h}$ (Fig. 1). The control strains S. aureus TX0117, ATCC 29213, and ATCC 25923 were included in the standardization of the test. Development and rapid test validation were performed in parallel in both the Colombian and U.S. laboratories.

Statistical analysis. We initially evaluated the sensitivity and specificity of the complete data set and, subsequently, calculations were made based on BlaZ types and the MIC-HI using MedCalc for Windows, version 15.0 (MedCalc Software, Ostend, Belgium). We used published prevalence ranges to calculate positive and negative predictive values (PPV and NPV, respectively) in each country (35). A receiver operating characteristic (ROC) curve for predictions of diagnostic performance was constructed using the complete data set of MSSA isolates and the Scikit-learn (36) module for Python programming language. The area under the curve (AUC) and 95\% confidence interval (CI) of the AUC were calculated.

Data availability. The genomes of the Latin American and U.S. isolates were submitted to the NCBI GenBank database under the BioProject numbers PRJNA580194, PRJNA595347, PRJNA291213 and PRJNA648430.

\section{RESULTS}

Development and validation of the CzIE rapid test. The CZIE rapid test was validated in 689 MSSA bloodstream isolates recovered from Latin America $(n=640)$ and the United States $(n=49)$ (Table 1). Overall, 257 (37\%) MSSA isolates exhibited CzIE. The CzIE in MSSA from Latin America was 40\% (range $31 \%$ to $55 \%$ ), and $10 \%$ from 
TABLE 1 Diagnostic performance of the rapid test for detection of the CzIE in MSSA isolates recovered from selected hospitals in Latin America and the United States

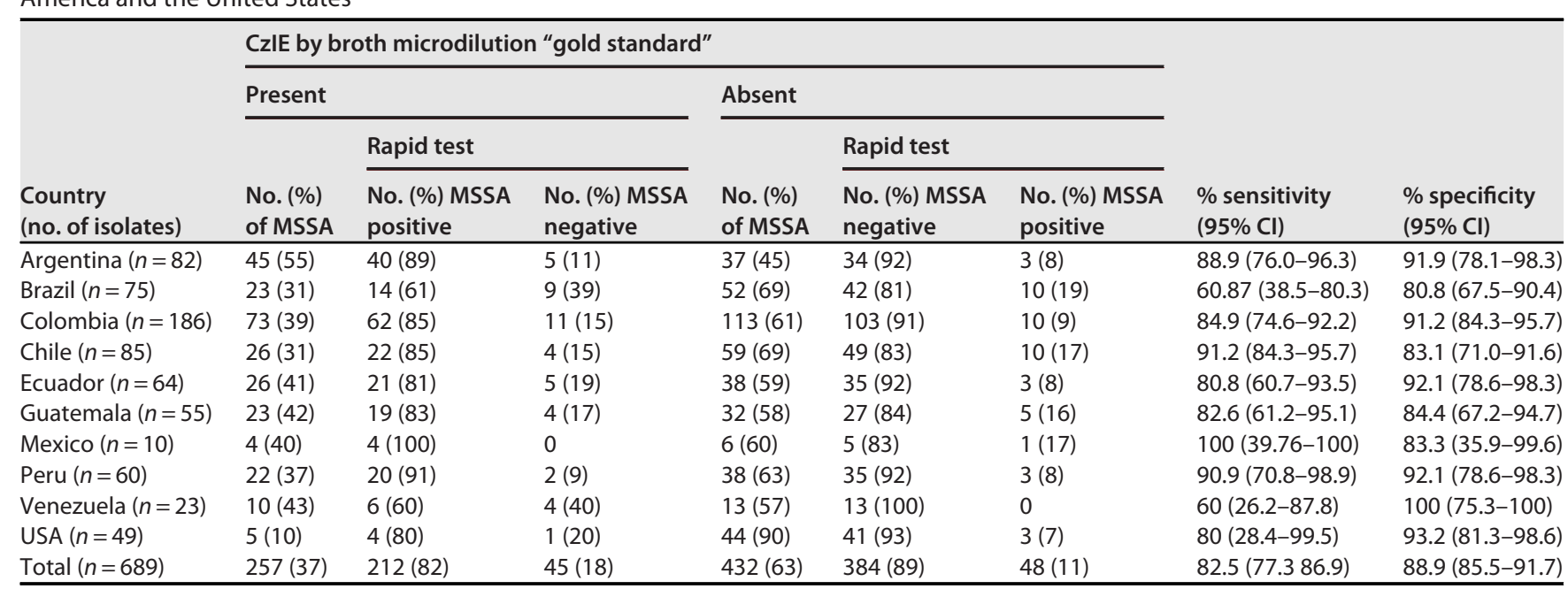

archived U.S. isolates (Table 1). The global sensitivity of the test was $82.5 \%$, with a specificity of $88.9 \%$ (Table 1). The diagnostic performance of the CzIE rapid test showed variation among countries. For example, Colombia provided the highest number of strains $(n=186)$ and $39 \%$ exhibited the CzIE. The sensitivity and specificity of the CzIE rapid test when applied to MSSA isolates from Colombia was $84.9 \%$ and 91.2\%, respectively. In contrast, Venezuela provided 23 MSSA and 10 (43\%) exhibited CzIE. In these isolates, the rapid test had a low sensitivity (60\%) but a high specificity (100\%). Of note, in 49 archived isolates available from United States, only 5 were positive for CzIE. The sensitivity and specificity of the test was $80 \%$ and $93.2 \%$, respectively (Table 1).

Accuracy of the CzIE rapid test varies with the type of $\boldsymbol{\beta}$-lactamase. The CzIE has been associated with the presence of staphylococcal $\beta$-lactamases type $A$ and $C(13$, 16-23). The type A enzyme seems to have enhanced activity against nitrocefin and cefazolin, whereas type $C$ has more affinity for cephalothin than cefazolin (37). Of note, BlaZ type $C$ was the most common in MSSA bloodstream isolates included in the study, followed by type A, with variations observed across the countries (Fig. S3). Indeed, when we evaluated the performance of the rapid test stratified by $\beta$-lactamase type, the test performed better in detecting the CzIE in MSSA isolates that harbored type A BlaZ than other types (Table 2), with sensitivity and specificity of $92.7 \%$ and $87.8 \%$, respectively. For type $C$, sensitivity and specificity were lower $(72.3 \%$ and $82.7 \%$, respectively), consistent with the decreased ability of type C enzyme to hydrolyze nitrocefin (37). The lower performance of the test against enzymes that do not have a robust ability to hydrolyze nitrocefin was evident when evaluating its performance against MSSA isolates harboring type B $\beta$-lactamase (37) (sensitivity of $53.3 \%$ ). Of note, the rapid test exhibited $100 \%$ specificity against strains that lack blaZ (Table 2).

The receiver operating characteristic (ROC) analysis for the whole data set of MSSA isolates and the overall AUC of 0.85 ( $95 \% \mathrm{Cl}: 0.83$ to 0.88$)$ indicated a good diagnostic accuracy of the CzIE rapid test (Fig. 2). Likewise, the tests exhibited an AUC value of 0.90 ( $95 \% \mathrm{Cl}: 0.85$ to 0.95 ) for BlaZ type A isolates, supporting the robust performance of the test against MSSA harboring staphylococcal type A enzymes.

Recently, some particular allotypes of BlaZ were associated with the CzIE in clinical isolates of MSSA recovered from Latin America (23). However, when we analyzed discrepant results of the rapid test (false positives and false negatives) to determine whether there was association with BlaZ allotypes (Table S1), we did not find any correlation. 
TABLE 2 Performance of the CzIE rapid test stratified by type of $\beta$-lactamase

\begin{tabular}{|c|c|c|c|c|c|}
\hline \multirow{2}{*}{$\begin{array}{l}\text { S. aureus tested } \\
\text { (no. of isolates) }\end{array}$} & \multirow{2}{*}{$\begin{array}{l}\text { Nitrocefin } \\
\text { rapid test } \\
\text { result }\end{array}$} & \multicolumn{2}{|c|}{$\begin{array}{l}\text { CzIE MIC by gold } \\
\text { standard }^{a}\end{array}$} & \multirow{2}{*}{$\begin{array}{l}\% \text { sensitivity } \\
(95 \% \mathrm{Cl})^{a}\end{array}$} & \multirow{2}{*}{$\begin{array}{l}\% \text { specificity } \\
(95 \% \mathrm{Cl})^{a}\end{array}$} \\
\hline & & Presence & Absence & & \\
\hline \multirow[t]{3}{*}{ Total of MSSA $(n=689)$} & Positive & 212 & 48 & $82.5(77.3-86.9)$ & $88.9(85.5-91.7)$ \\
\hline & Negative & 45 & 384 & & \\
\hline & Total & 257 & 432 & & \\
\hline \multirow[t]{3}{*}{ BlaZ type $\mathrm{A}(n=186)$} & Positive & 127 & 6 & $92.7(87-96.4)$ & $87.8(75.2-95.4)$ \\
\hline & Negative & 10 & 43 & & \\
\hline & Total & 137 & 49 & & \\
\hline \multirow[t]{3}{*}{ BlaZ type B $(n=157)$} & Positive & 8 & 24 & $53.3(26.6-78.7)$ & $83.1(75.9-88.9)$ \\
\hline & Negative & 7 & 118 & & \\
\hline & Total & 15 & 142 & & \\
\hline \multirow[t]{3}{*}{ BlaZ type C $(n=205)$} & Positive & 73 & 18 & $72.3(62.5-80.7)$ & $82.7(74-89.4)$ \\
\hline & Negative & 28 & 86 & & \\
\hline & Total & 101 & 104 & & \\
\hline \multirow[t]{3}{*}{ BlaZ type $\mathrm{D}(n=5)$} & Positive & 4 & 0 & NA & NA \\
\hline & Negative & 0 & 1 & & \\
\hline & Total & 4 & 1 & & \\
\hline \multirow[t]{3}{*}{ BlaZ nontypeable $(n=1)$} & Positive & 0 & 0 & NA & NA \\
\hline & Negative & 0 & 1 & & \\
\hline & Total & 0 & 1 & & \\
\hline \multirow[t]{3}{*}{ BlaZ negative $(n=135)$} & Positive & 0 & 0 & NA & 100 \\
\hline & Negative & 0 & 135 & & \\
\hline & Total & 0 & 135 & & \\
\hline
\end{tabular}

${ }^{a} \mathrm{CzIE}$, cefazolin inoculum effect, identified by broth microdilution at high inoculum; $\mathrm{Cl}$, confidence interval; $\mathrm{NA}$, not applicable.

${ }^{b}$ The nontypeable BlaZ indicates the presence of lysine (K) at position 119 and serine (S) at amino acid position 207 in the BlaZ protein (9).

Correlation between the CzIE rapid test and the cefazolin MICs at high inoculum. Among MSSA isolates harboring type A and type C BlaZ, 137/186 (74\%) and 101/205 (49\%) exhibited the CzIE, respectively, using the gold standard MIC determination (high inoculum). We postulated that the CzIE was highly correlated with the

A

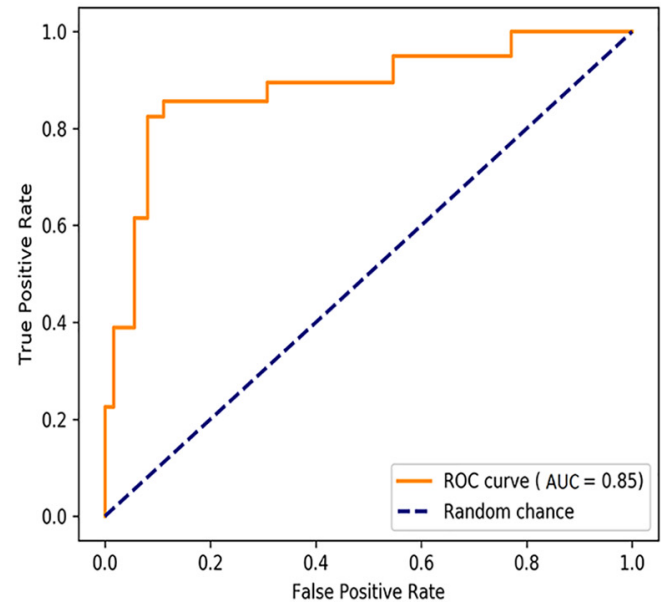

B

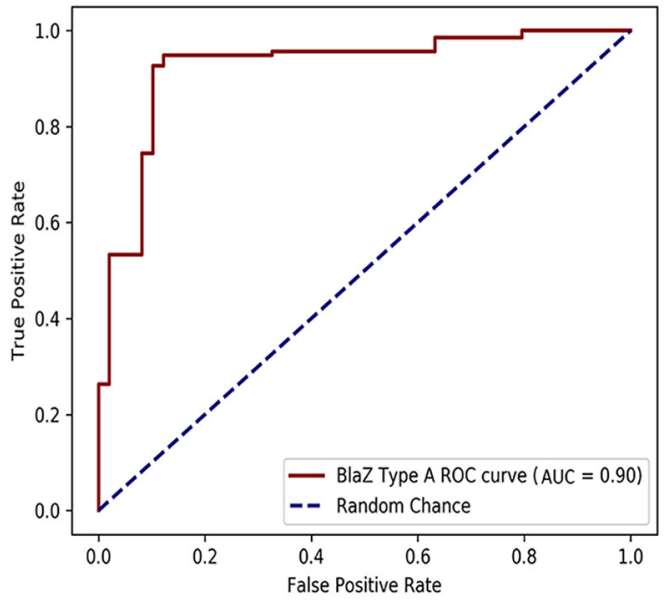

FIG 2 Receiver operational characteristic (ROC) curve and area under the ROC curve (AUC) to evaluate the diagnostic performance of the CzIE rapid test. (A) ROC curve for the complete set of MSSA isolates $(n=689)$. (B) ROC curves for BlaZ type A isolates $(n=186)$. The dotted lines represent random chance (AUC $=0.5$ ). 
TABLE 3 Performance of the CzIE rapid test according to $\beta$-lactamase type and high inoculum MIC

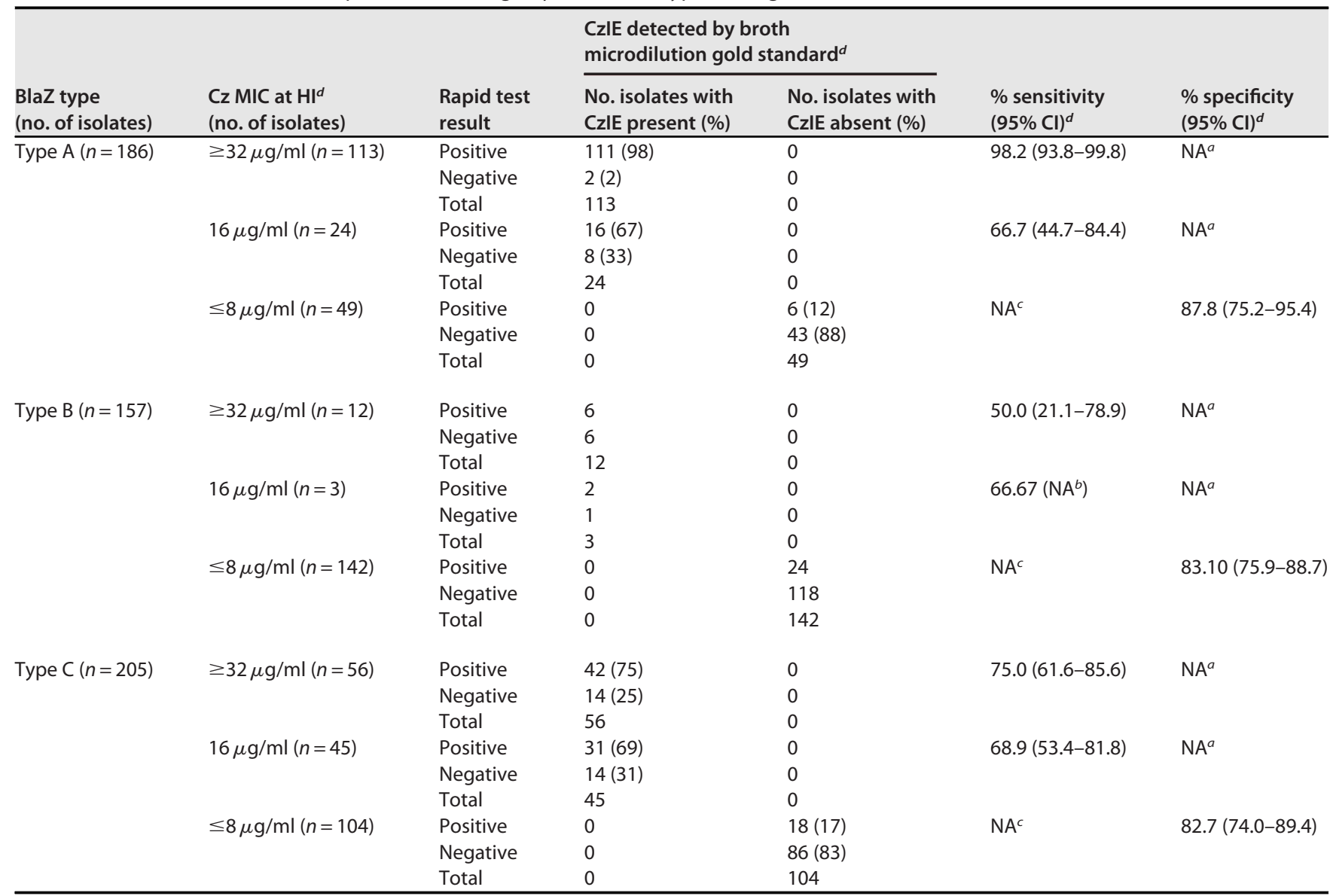

${ }^{a}$ Not applicable due to inability to estimate the value. To calculate the specificity, the number of false positives (FP) and number of true negatives (TN) derived from the column that indicates "absence of the CZIE" are required. Therefore, we were unable to estimate this value since FP=0 and TN $=0$.

bNot applicable due to small sample size.

cNot applicable due to the inability to estimate the value. To calculate the sensitivity, the number of true positive (TP) and number of false negative (FN) derived from the column that indicates "presence of the CZIE" are required. Therefore, in these cases we were unable to estimate this value since TP $=0$ and $\mathrm{FN}=0$.

${ }^{d} \mathrm{CZIE}$, cefazolin inoculum effect, identified by broth microdilution at high inocula ( $\left.10^{7} \mathrm{CFU} / \mathrm{ml}\right)$; $\mathrm{Cl}$, confidence interval; $\mathrm{HI}$, high inoculum.

extracellular amount of the enzyme and that isolates with higher cefazolin MIC-HI are most likely to fail therapy with cefazolin. Our cutoff for determining the CzIE effect was $\geq 16 \mu \mathrm{g} / \mathrm{ml}$ based on previous analyses that suggested this value was the lowest MIC likely to correlate with poor clinical outcomes (13). Thus, we evaluated the performance of the CZIE rapid test stratified by MIC-HI. Table 3 shows that the sensitivity of the test markedly increased (98.2\%) in isolates with type A BlaZ that exhibit MICs of $\geq 32 \mu \mathrm{g} / \mathrm{ml}$, suggesting that the test would be reliable in strains that release robust amounts of type A enzyme.

Positive and negative predictive values of the CzIE rapid test. We estimated the effectiveness of the rapid test using positive and negative predictive values (PPV and NPV, respectively) (35). Since there are limited data on the prevalence of the CzIE in MSSA, we incorporated published data available for bloodstream isolates from participating countries $(13,19,21-23)$, contributing $>10$ isolates. Table 4 shows that the NPV ranged from $81.4 \%$ to $95.6 \%$ overall, whereas the PPV ranged from $59.8 \%$ to $100 \%$ in Latin American countries. In the United States, few studies have investigated the frequency of CzIE in MSSA from different clinical sources, with wide variations in prevalence estimates of the CzIE (19-21). Due to the limited number of isolates available from the United States, we used different estimates of prevalence. Using prevalence estimates ranging from $25 \%$ to $4 \%$, respectively, the NPV ranged from $93.3 \%$ to $97.7 \%$. In contrast, the PPV exhibited wide variations (from $32.8 \%$ to $79.6 \%$ ) (Table 4). 
TABLE 4 Positive and negative predicted values of the rapid test according to the reported prevalence of the CzIE in bloodstream MSSA from Latin America and the United States

\begin{tabular}{lllllll}
\hline Country & \% Prevalence $^{a}$ & Sensitivity $^{b}$ & Specificity $^{b}$ & PPV $^{c}$ & NPV $^{c}$ & Source $^{\text {Spentina }}$ \\
\hline Argentl & 55 & 88.9 & 91.9 & 92.8 & 87.6 & $(13)$ \\
Brazil & 32 & 60.9 & 80.8 & 59.8 & 81.4 & $(23)$ \\
Colombia & $39^{d}$ & 84.9 & 91.2 & 86.0 & 90.4 & $(22,23)$ \\
Chile & 32 & 91.2 & 83.1 & 70.1 & 92.0 & $(23)$ \\
Ecuador & $41^{d}$ & 80.8 & 92.1 & 87.7 & 87.3 & $(22,23)$ \\
Guatemala & 44 & 82.6 & 84.4 & 80.6 & 86.1 & $(23)$ \\
Perú & $32^{d}$ & 90.9 & 92.1 & 84.4 & 95.6 & $(22,23)$ \\
Venezuela & $30.5^{d}$ & 60 & 100 & 100 & 85.1 & $(22,23)$ \\
United States & 25 & 80.0 & 93.2 & 79.6 & 93.3 & $(21)^{e}$ \\
& 4 & 80.0 & 93.2 & 32.8 & 99.1 & $(19)$ \\
& 10 & 80.0 & 93.2 & 56.6 & 97.7 & This study \\
\hline
\end{tabular}

aprevalence of the CzIE in MSSA isolates recovered from bloodstream infections in each country, as reported previously. Only prevalences based on cefazolin MIC $\geq 16 \mu \mathrm{g} / \mathrm{ml}$ at high inoculum were included.

bSensitivity and specificity of the rapid test calculated in this study.

cPositive predictive values (PPV) and negative predictive values (NPV) were calculated taking into consideration previous reported prevalences.

'The value represents prevalence mean of two reported studies.

${ }^{e}$ The prevalence of CzIE in this study correspond to isolates recovered from 24 hemodialysis patients.

Phylogenetic analyses of U.S. MSSA and the CzIE. Our recently published data showed that bloodstream MSSA isolates from Latin America belonged to a variety of genetic lineages and that CC30 was the predominant lineage among MSSA exhibiting the CzIE (23). Here, we reconstructed the phylogenetic tree using core genes of Latin American strains (23) and included U.S. isolates. Similar to our previous findings in Latin America, we show an important genetic heterogeneity among U.S. MSSA, but no distinct genetic differences between U.S. and Latin American MSSA isolates (Fig. S2). The 49 U.S. isolates were distributed in $18 \mathrm{STs}$ and the most common clonal lineage was CC5 $(28 \%, n=14)$, followed by CC15 $(12 \%, n=6)$ and CC8 $(10 \%, n=5)$.

\section{DISCUSSION}

We have developed a novel, rapid colorimetric test to identify the presence of CzIE in clinical isolates of MSSA. Although the impact of CzIE on the outcome of MSSAinfected patients treated with cefazolin is incompletely understood, a growing number of studies $(13,15)$ have reported an association of the CzIE with increased mortality and worse outcomes. These data are supported by animal models that confirm the CzIE leads to cefazolin treatment failure (38). In this context, it seems reasonable to assume that identifying the CzIE may be a useful tool when treating severe MSSA infections with cefazolin. However, detecting the CzIE is not a trivial task. While the "gold standard" for the CzIE detection is to perform high-inoculum MIC determinations, there are no current standardized guidelines for the execution of this method, resulting in the utilization of additional personnel, resources, and time (i.e., MIC determinations at high inoculum size usually should be performed in triplicates), and requiring expertise in interpretation of results. Furthermore, results are only available after 2 to 3 days and the test requires highly trained personnel. In consequence, the gold standard would not be readily adopted in the clinical microbiology workflow. Therefore, developing a reliable and low-cost rapid test to identify MSSA isolates exhibiting the CzIE could potentially be a major advantage when making therapeutic decisions for MSSA infections.

Expression, processing, and extracellular release of BlaZ in S. aureus is complex and requires the participation of multiple enzymes (24). Here, we present a novel nitrocefin-based colorimetric test that was developed based on the observation that detection of the extracellular $\beta$-lactamase activity (as opposed to membrane-bound $\beta$-lactamase) in supernatants of MSSA strains correlated with the presence of CzIE in prototypical strains (Fig. S1). Nitrocefin has been broadly used for detection and 
characterization of S. aureus $\beta$-lactamase (BlaZ) $(39,40)$. Furthermore, the profile of nitrocefin breakdown has been key for the classification of BlaZ into the different types (A, B, C, or D) (37). Although routine nitrocefin disk testing is unable to discriminate between released and membrane-bound $\beta$-lactamase, when we evaluated disks with bacterial supernatants (after induction with ampicillin), we noted that the nitrocefin seem to work well with control strains but did not perform properly when a larger collection of isolates (previously characterized for the CZIE) was tested. In contrast, nitrocefin solution permitted a proper visualization of the enzymatic activity of released BlaZ in these isolates and, therefore, was used as the preferred colorimetric substrate to develop the rapid test. Furthermore, in the process of test development, we used control strains to assess different time points. In the validation process with a large set of isolates, we noted that those isolates exhibiting the CzIE but harboring enzymes with less affinity to cefazolin could be detected if the period was extended to $2 \mathrm{~h}$. After $2 \mathrm{~h}$, the negative controls also turned color and could no longer be discriminated. Therefore, based on these observations, we decided to extend the reading of the rapid test to up to $2 \mathrm{~h}$. Subsequently, using our robust collection of MSSA isolates from Latin America and the United States characterized for the $\mathrm{CZIE}$, we aimed to validate the rapid colorimetric CzIE test.

Interestingly, the overall prevalence of the CzIE was 37\%, ranging from $10 \%$ (U.S.) to $55 \%$, although U.S. isolates were mainly from archived isolates. This is an important issue since the use of cefazolin as first line therapy for MSSA has increased in the last few years. Thus, it is possible that the frequencies reported here, especially among U.S. isolates, may be underestimated. The overall sensitivity and specificity of the test were $82.5 \%$ and $88.9 \%$, respectively. The availability of whole-genome sequencing in all of our isolates allowed us to identify the most common BlaZ types associated with CzIE. As expected, the accuracy of the CzIE rapid test varied with the type of $\beta$-lactamase. Type $A$ is by far the most efficient in hydrolyzing cefazolin and nitrocefin compared to type $C$ or type $B$ (37). Thus, it is not surprising that the test performs much better in isolates that harbor type A BlaZ, with sensitivity and specificity of $92.7 \%$ and $87.8 \%$, respectively. In contrast, the test sensitivity in isolates with type $C$ and $B$ was less robust $(72.3 \%$ and $53.3 \%$, respectively). The lower performance is likely related to the poorer activity of type $B$ and $C$ enzymes against nitrocefin. However, we noted that in certain countries with high prevalence of type $B$ and $C$ enzymes, the test still performed well (e.g., Guatemala, Table 1 and Fig. S3). To explain this phenomenon, we recently showed (23) that there is much more variation in BlaZ sequences than previously thought. Indeed, within the same type, there were many variants (allotypes) that may have differential activity against nitrocefin. Thus, we analyzed all cases of false positives and negatives to evaluate if an association of performance of the CzIE could be identified with any particular allotype. However, we were not able to identify any individual allotype association (Table S1). Thus, our results suggest that the variability in the performance of the test seen in strains with the same BlaZ types could be due in part to kinetic differences between the allotypes that belong to the same BlaZ type. Moreover, our results suggest that unidentified variations between strain backgrounds may impact the performance of the test. Furthermore, the type of enzyme circulating in each country is likely to be determined by the type of cephalosporin used in therapy (e.g., cefazolin versus cephalothin).

We also carried out an analysis of test performance based on the MIC-HI. The cutoff of $16 \mu \mathrm{g} / \mathrm{ml}$ of cefazolin was chosen based on our previous data showing that this MIC value correlates with mortality in patients with bacteremia (13), and our initial results indicated that $62 \%$ of false negatives exhibited MICs close to the cutoff value. Thus, we investigated the performance of the CzIE in isolates with MICs of $\geq 32 \mu \mathrm{g} / \mathrm{ml}$, which are more likely to exhibit a strong CzIE and also cause therapeutic problems with cefazolin, since we assume they release larger amounts of the enzyme into the extracellular milieu. Thus, by evaluating an alternative cutoff value of $\geq 32 \mu \mathrm{g} / \mathrm{ml}$, the sensitivity of the CzIE increased to $98.2 \%$ for type A enzymes, suggesting that the test would be able to 
identify most isolates with a robust $\mathrm{CzIE}$, which could be the ones with the greatest clinical impact. Of note, the specificity of the rapid CZIE test was $100 \%$ in isolates that lacked blaZ.

We also calculated the PPV and NPV values using published and estimated prevalences of the $\mathrm{CZIE}$, as it was difficult to determine the overall prevalence of this phenomenon in the participating countries. We believe that, in the context of CzIE, high sensitivity and NPV would be the most informative characteristics of a test to aid in therapeutic decisions. Our estimates suggest that the test has a good NPV at low and high prevalence (Table 4).

Our study has several limitations. First, the isolates included in the cohort are mainly from Latin America (93\%) and, thus, measures of performance of the test could vary when applied to isolates from other geographical locations, as the pretest probability would be influenced by the prevalence of the CzIE in each region. Second, the test was developed in a research laboratory and real-world performance of the test needs to be validated. Third, only isolates causing bloodstream infections were included in this cohort, and it is unclear if the results would apply to isolates recovered from other infections. Finally, we did not assess clinical outcomes in the present cohort due to the lack of reliable information in all patients and it is beyond the scope of this report. We used a cutoff value of $\geq 16 \mu \mathrm{g} / \mathrm{ml}$ that was previously associated with increased mortality in MSSA bacteremia (13).

In summary, we have developed a simple, inexpensive, and novel nitrocefin-based test to identify MSSA isolates exhibiting CzIE. This test would provide important information that is not currently available in any clinical microbiology laboratory with results available in $3 \mathrm{~h}$. The test is easy to perform and does not require specialized equipment or reagents and interpretation is straightforward, suggesting lower barriers to implementation and suitability even in resource-limited locations.

\section{SUPPLEMENTAL MATERIAL}

Supplemental material is available online only.

SUPPLEMENTAL FILE 1, PDF file, $0.1 \mathrm{MB}$.

SUPPLEMENTAL FILE 2, PDF file, $0.3 \mathrm{MB}$.

SUPPLEMENTAL FILE 3, PDF file, $0.1 \mathrm{MB}$.

SUPPLEMENTAL FILE 4, PDF file, $0.04 \mathrm{MB}$.

\section{ACKNOWLEDGMENTS}

We are grateful to the following investigators who provided the isolates in the participating hospitals: Mauro J. Salles, Division of Infectious Diseases, Department of Internal Medicine, Santa Casa de Sao Paulo School of Medicine, Sao Paulo, Brazil; Carlos Alvarez-Moreno, Unidad Infectologia, Departamento de Medicina Interna, Facultad de Medicina, Universidad Nacional de Colombia, Clinica Universitaria Colombia, Colsanitas, Bogota, Colombia; Jaime Labarca, Department of Infectious Diseases, School of Medicine, Pontificia Universidad Catolica de Chile, Santiago, Chile; Coralith Garcia, Hospital Cayetano Heredia, Instituto de Medicina Tropical Alexander von Humboldt, Universidad Peruana Cayetano Heredia, Lima, Peru; Carlos M. Luna, Pulmonary Division, Department of Medicine, Jose de San Martin Hospital, University of Buenos Aires, Buenos Aires, Argentina; Carlos Mejia-Villatoro, Clinica de Enfermedades Infecciosas, Hospital Roosevelt, Guatemala City, Guatemala; Jeannete Zurita, Hospital Vozandes, Facultad de Medicina, Pontificia Universidad Catolica del Ecuador, Quito, Ecuador; Manuel Guzman-Blanco, Centro Medico de Caracas, Caracas, Venezuela; Eduardo Rodriguez-Noriega, Hospital Civil de Guadalajara, Fray Antonio Alcalde, and Instituto de Patologia Infecciosa y Experimental, Centro Universitario de Ciencias de la Salud, Universidad de Guadalajara, Guadalajara, Mexico.

We thank Jessica Porras, Sandra Vargas, Catalina Espitia, Karina Torres, and Oscar Ortega for their technical support.

This work was supported by funding from Colciencias-MinCiencias (grant 130880764150 awarded to S.R.) and National Institutes of Health (NIH) NIAID (grant R21 AI143229 awarded to C.A.A.). J.R. is supported by Colciencias-MinCiencias grant 130880764152 and L.D. is 
supported by Colciencias-MinCiencias grant 130874455850 . C.A.A. is also supported by $\mathrm{NIH} /$ NIAID grants R01 Al134637, R01 Al148342, and K24 Al121296, UTHealth Presidential Award and University of Texas System STARS Award. B.E.M. was supported in part by NIH grant R21 Al133289. W.R.M. is supported by NIH/NIAID grant K08 Al135093. T.P. is supported by NIH/ NIAID AI32956. The funders had no role in study design, data collection and interpretation, or the decision to submit the work for publication.

C.A.A. has received grant support from Merck, Inc, Entasis Therapeutics and MeMed diagnostics. E.N. has received grants from Pfizer SRL Janssen and Basilea Pharmaceutica and personal fees from Merck Sharp \& Dohme and Pfizer. V.G.F. reports grant/research support from the National Institutes of Health, Medlmmune, Cerexa/Forest/Actavis/ Allergan, Pfizer, Advanced Liquid Logics, Theravance, Novartis, Cubist/Merc, Medical Biosurfaces, Locus, Affinergy, Contrafect, Karius, Genentech, Regeneron, and Basilea, and has worked as a paid consultant for Pfizer, Novartis, Galderma, Novadigm, Durata, Debiopharm, Genentech, Amphliphi Biosciences, Integrated Biotherapeutics, C3J, Achaogen, Affinium, Medicines Co., Cerexa, Tetraphase, Trius, Medlmmune, Bayer, Theravance, Cubist, Basilea, Affinergy, Janssen, xBiotech, Contrafect, Regeneron, Basilea, and Destiny. V.G.F was also a member of Merck Co-Chair V710 Vaccine and received educational fees from Green Cross, Cubist, Cerexa, Durata, Theravance, and Debiopharm, as well as royalties from UpToDate. He has a patent pending for host gene expression signature diagnostic for sepsis. W.R.M. has received grants and/or honoraria from Merck, Entasis Therapeutics, and Shionogi. The rest of the authors report no conflicts of interest. All authors have submitted the ICMJE Form for Disclosure of Potential Conflicts of Interest.

\section{REFERENCES}

1. Arias CA, Reyes J, Carvajal LP, Rincon S, Diaz L, Panesso D, Ibarra G, Rios R, Munita JM, Salles MJ, Alvarez-Moreno C, Labarca J, Garcia C, Luna CM, Mejia-Villatoro C, Zurita J, Guzman-Blanco M, Rodriguez-Noriega E, Narechania A, Rojas LJ, Planet PJ, Weinstock GM, Gotuzzo E, Seas C. 2017. A prospective cohort multicenter study of molecular epidemiology and phylogenomics of Staphylococcus aureus bacteremia in nine Latin American countries. Antimicrob Agents Chemother 61:e00816-17. https://doi .org/10.1128/AAC.00816-17.

2. Hadler JL, Petit S, Mandour M, Cartter ML. 2012. Trends in invasive infection with methicillin-resistant Staphylococcus aureus, Connecticut, USA, 2001-2010. Emerg Infect Dis 18:917-924. https://doi.org/10.3201/eid1806 .120182.

3. Jernigan JA, Hatfield KM, Wolford H, Nelson RE, Olubajo B, Reddy SC, McCarthy N, Paul P, McDonald LC, Kallen A, Fiore A, Craig M, Baggs J. 2020. Multidrug-resistant bacterial infections in U.S. hospitalized patients, 2012-2017. N Engl J Med 382:1309-1319. https://doi.org/10.1056/ NEJMoa1914433.

4. Jackson KA, Gokhale RH, Nadle J, Ray SM, Dumyati G, Schaffner W, Ham DC, Magill SS, Lynfield R, See I. 2020. Public health importance of invasive Methicillin-sensitive Staphylococcus aureus infections: surveillance in 8 US counties, 2016. Clin Infect Dis 70:1021-1028. https://doi.org/10.1093/cid/ ciz323.

5. Loubet P, Burdet C, Vindrios W, Grall N, Wolff M, Yazdanpanah Y, Andremont A, Duval X, Lescure FX. 2018. Cefazolin versus anti-staphylococcal penicillins for treatment of methicillin-susceptible Staphylococcus aureus bacteraemia: a narrative review. Clin Microbiol Infect 24:125-132. https://doi.org/10.1016/j.cmi.2017.07.003.

6. Flynt LK, Kenney RM, Zervos MJ, Davis SL. 2017. The safety and economic impact of cefazolin versus nafcillin for the treatment of methicillin-susceptible Staphylococcus aureus bloodstream infections. Infect Dis Ther 6:225-231. https://doi.org/10.1007/s40121-017-0148-z.

7. McDanel JS, Roghmann M-C, Perencevich EN, Ohl ME, Goto M, Livorsi DJ, Jones M, Albertson JP, Nair R, O'Shea AMJ, Schweizer ML. 2017. Comparative effectiveness of Cefazolin versus Nafcillin or Oxacillin for treatment of Methicillin-susceptible Staphylococcus aureus infections complicated by bacteremia: a nationwide cohort study. Clin Infect Dis 65:100-106. https:// doi.org/10.1093/cid/cix287.

8. Youngster I, Shenoy ES, Hooper DC, Nelson SB. 2014. Comparative evaluation of the tolerability of cefazolin and nafcillin for treatment of methicillin-susceptible Staphylococcus aureus infections in the outpatient setting. Clin Infect Dis 59:369-375. https://doi.org/10.1093/cid/ciu301.

9. Bai AD, Showler A, Burry L, Steinberg M, Ricciuto DR, Fernandes T, Chiu A, Raybardhan S, Science M, Fernando E, Tomlinson G, Bell CM, Morris AM. 2015. Comparative effectiveness of cefazolin versus cloxacillin as definitive antibiotic therapy for MSSA bacteraemia: results from a large multicentre cohort study. J Antimicrob Chemother 70:1539-1546. https://doi .org/10.1093/jac/dku560.

10. Rao SN, Rhodes NJ, Lee BJ, Scheetz MH, Hanson AP, Segreti J, Crank CW, Wang SK. 2015. Treatment outcomes with cefazolin versus oxacillin for deep-seated methicillin-susceptible Staphylococcus aureus bloodstream infections. Antimicrob Agents Chemother 59:5232-5238. https://doi.org/ 10.1128/AAC.04677-14.

11. Li J, Echevarria KL, Traugott KA. 2017. $\beta$-Lactam therapy for Methicillin-susceptible Staphylococcus aureus bacteremia: a comparative review of Cefazolin versus antistaphylococcal penicillins. Pharmacotherapy 37:346-360. https://doi.org/10.1002/phar.1892.

12. Pollett S, Baxi SM, Rutherford GW, Doernberg SB, Bacchetti $P$, Chambers HF. 2016. Cefazolin versus nafcillin for methicillin-sensitive Staphylococcus aureus bloodstream infection in a California tertiary medical center. Antimicrob Agents Chemother 60:4684-4689. https://doi.org/10.1128/AAC .00243-16.

13. Miller WR, Seas C, Carvajal LP, Diaz L, Echeverri AM, Ferro C, Rios R, Porras P, Luna C, Gotuzzo E, Munita JM, Nannini E, Carcamo C, Reyes J, Arias CA. 2018. The Cefazolin inoculum effect is associated with increased mortality in Methicillin-susceptible Staphylococcus aureus bacteremia. Open Forum Infect Dis 5:ofy123. https://doi.org/10.1093/ofid/ofy123.

14. Nannini EC, Singh KV, Murray BE. 2003. Relapse of type A $\beta$-lactamaseproducing Staphylococcus aureus native valve endocarditis during cefazolin therapy: revisiting the issue. Clin Infect Dis 37:1194-1198. https://doi .org/10.1086/379021.

15. Lee S, Song KH, Jung SI, Park WB, Lee SH, Kim YS, Kwak YG, Kim YK, Kiem SM, Kim HI, Kim ES, Park KH, Kim NJ, Jang HC, Kim HB, Korea INfectious Diseases (KIND) study group. 2018. Comparative outcomes of cefazolin versus nafcillin for methicillin-susceptible Staphylococcus aureus bacteraemia: a prospective multicentre cohort study in Korea. Clin Microbiol Infect 24:152-158. https://doi.org/10.1016/j.cmi.2017.07.001.

16. Lee SO, Lee S, Park S, Lee JE, Lee SH. 2019. The cefazolin inoculum effect and the presence of type A blaZ gene according to agr genotype in 
methicillin-susceptible Staphylococcus aureus bacteremia. Infect Chemother 51:376-385. https://doi.org/10.3947/ic.2019.51.4.376.

17. Chong YP, Park S, Kim ES, Bang KM, Kim MN, Kim SH, Lee SO, Choi SH, Jeong JY, Woo JH, Kim YS. 2015. Prevalence of blaZ gene types and the cefazolin inoculum effect among methicillin-susceptible Staphylococcus aureus blood isolates and their association with multilocus sequence types and clinical outcome. Eur J Clin Microbiol Infect Dis 34:349-355. https://doi.org/10.1007/s10096-014-2241-5.

18. Saeki M, Shinagawa M, Yakuwa Y, Nirasawa S, Sato Y, Yanagihara N, Takahashi S. 2018. Inoculum effect of high concentrations of methicillinsusceptible Staphylococcus aureus on the efficacy of cefazolin and other beta-lactams. J Infect Chemother 24:212-215. https://doi.org/10.1016/j .jiac.2017.10.021.

19. Livorsi DJ, Crispell E, Satola SW, Burd EM, Jerris M, Wang YF, Farley MM. 2012. Prevalence of blaZ gene types and the inoculum effect with cefazolin among bloodstream isolates of methicillin-susceptible Staphylococcus aureus. Antimicrob Agents Chemother 56:4474-4477. https://doi.org/10 .1128/AAC.00052-12.

20. Wang SK, Gilchrist A, Loukitcheva A, Plotkin BJ, Sigar IM, Gross AE, O'Donnell JN, Pettit N, Buros A, O'Driscoll T, Rhodes NJ, Bethel C, Segreti J, CharnotKatsikas A, Singh K, Scheetz MH. 2018. Prevalence of a cefazolin inoculum effect associated with blaZ gene types among methicillin-susceptible Staphylococcus aureus isolates from four major medical centers in Chicago. Antimicrob Agents Chemother 62:e00382-18. https://doi.org/10 .1128/AAC.00382-18.

21. Nannini EC, Stryjewski ME, Singh KV, Bourgogne A, Rude TH, Corey GR, Fowler VG, Jr, Murray BE. 2009. Inoculum effect with cefazolin among clinical isolates of methicillin-susceptible Staphylococcus aureus: frequency and possible cause of cefazolin treatment failure. Antimicrob Agents Chemother 53:3437-3441. https://doi.org/10.1128/AAC.00317-09.

22. Rincon S, Reyes J, Carvajal LP, Rojas N, Cortes F, Panesso D, Guzmán M, Zurita J, Adachi JA, Murray BE, Nannini EC, Arias CA. 2013. Cefazolin highinoculum effect in methicillin-susceptible Staphylococcus aureus from South American hospitals. J Antimicrob Chemother 68:2773-2778. https:// doi.org/10.1093/jac/dkt254.

23. Carvajal LP, Rincon S, Echeverri AM, Porras J, Rios R, Ordoñez KM, Seas C, Gomez-Villegas SI, Diaz L, Arias CA, Reyes J. 2020. Novel insights into the classification of staphylococcal $\beta$-lactamases in relation to the cefazolin inoculum effect. Antimicrob Agents Chemother 64:e02511-19. https://doi .org/10.1128/AAC.02511-19.

24. Livermore DM. 1995. $\beta$-Lactamases in laboratory and clinical resistance. Clin Microbiol Rev 8:557-584. https://doi.org/10.1128/CMR.8.4.557-584 .1995 .

25. O'Callaghan CH, Morris A, Kirby SM, Shingler AH. 1972. Novel method for detection of beta-lactamases by using a chromogenic cephalosporin substrate. Antimicrob Agents Chemother 1:283-288. https://doi.org/10.1128/ aac. 1.4.283.

26. Carvajal LP, Santiago A, Echeverri AM, Rios R, Rincon S, Panesso D, Diaz L, Miller W, Sun Z, Palzkill T, Arias CA, Reyes J. 2018. Extracellular release of $\beta$-lactamase is responsible for the cefazolin inoculum effect (CzIE) in methicillin-susceptible Staphylococcus aureus. Open Forum Infect Dis 5:S602. https://doi.org/10.1093/ofid/ofy210.1719.

27. Voladri RK, Kernodle DS. 1998. Characterization of a chromosomal gene encoding type B beta-lactamase in phage group II isolates of Staphylococcus aureus. Antimicrob Agents Chemother 42:3163-3168. https://doi.org/ 10.1128/AAC.42.12.3163

28. Voladri RKR, Tummuru MKR, Kernodle DS. 1996. Structure-function relationships among wild-type variants of Staphylococcus aureus beta-lactamase: importance of amino acids 128 and 216. J Bacteriol 178:7248-7253. https://doi.org/10.1128/jb.178.24.7248-7253.1996.

29. Jolley KA, Maiden MCJ. 2010. BIGSdb: scalable analysis of bacterial genome variation at the population level. BMC Bioinformatics 11:595. https://doi.org/10.1186/1471-2105-11-595.

30. Page AJ, Cummins CA, Hunt M, Wong VK, Reuter S, Holden MT, Fookes M, Falush D, Keane JA, Parkhill J. 2015. Roary: rapid large-scale prokaryote pan genome analysis. Bioinformatics 31:3691-3693. https://doi.org/10 .1093/bioinformatics/btv421.

31. Edgar RC. 2004. MUSCLE: multiple sequence alignment with high accuracy and high throughput. Nucleic Acids Res 32:1792-1797. https://doi .org/10.1093/nar/gkh340.

32. Stamatakis A. 2014. RAxML version 8: a tool for phylogenetic analysis and post-analysis of large phylogenies. Bioinformatics 30:1312-1313. https:// doi.org/10.1093/bioinformatics/btu033.

33. Letunic I, Bork P. 2019. Interactive Tree Of Life (iTOL) v4: recent updates and new developments. Nucleic Acids Res 47:W256-W259. https://doi .org/10.1093/nar/gkz239.

34. National Committee for Clinical Laboratory Standards. 2012. Performance standards for antimicrobial susceptibility testing; twenty-second informational supplement.

35. Tenny S, Hoffman MR. 2010. Prevalence. StatPearls Publishing. https:// www.ncbi.nlm.nih.gov/books/NBK430867/. Accessed 26 July 2020.

36. Pedregosa F, Varoquaux G, Gramfort A, Michel V, Thirion B, Grisel O, Blondel M, Prettenhofer P, Weiss R, Dubourg V, Vanderplas J, Passos A, Cournapeau D, Brucher M, Perrot M, Duchesnay E. 2011. Scikit-learn: machine Learning in Python. J Mach Learn Res 12:2825-2830.

37. Zygmunt DJ, Stratton CW, Kernodle DS. 1992. Characterization of four beta-lactamases produced by Staphylococcus aureus. Antimicrob Agents Chemother 36:440-445. https://doi.org/10.1128/aac.36.2.440.

38. Singh KV, Tran TT, Nannini EC, Tam VH, Arias CA, Murray BE. 2017. Efficacy of ceftaroline in a rat model of endocarditis against methicillin-susceptible Staphylococcus aureus exhibiting the cefazolin high inoculum effect. Antimicrob Agents Chemother 61:e00324-17. https://doi.org/10.1128/ AAC.00324-17.

39. Takayama Y, Tanaka T, Oikawa K, Fukano N, Goto M, Takahashi T. 2018. Prevalence of blaZ gene and performance of phenotypic tests to detect penicillinase in Staphylococcus aureus isolates from Japan. Ann Lab Med 38:155-159. https://doi.org/10.3343/alm.2018.38.2.155.

40. Hombach M, Weissert C, Senn MM, Zbinden R. 2017. Comparison of phenotypic methods for the detection of penicillinase in Staphylococcus aureus and proposal of a practical diagnostic approach. J Antimicrob Chemother 72:1089-1093. https://doi.org/10.1093/jac/dkw521. 